\title{
A New Technique of Endoscopic Transpapillary Gallbladder Drainage Combined with Intraductal Ultrasonography for the Treatment of Acute Cholecystitis
}

\author{
Ryota Sagami', Kenji Hayasaka', Tetsuro Ujihara', Ryotaro Nakahara', Daisuke Murakami', Tomoyuki Iwaki ${ }^{1}$, Satoshi Suehiro', \\ Yasushi Katsuyama', Hideaki Harada' and Yuji Amano ${ }^{2}$ \\ Department of ${ }^{1}$ Gastroenterology, ${ }^{2}$ Endoscopy, New Tokyo Hospital, Chiba, Japan
}

Background/Aims: Endoscopic transpapillary gallbladder drainage (ETGBD) is useful for the treatment of acute cholecystitis; however, the technique is difficult to perform. When intraductal ultrasonography (IDUS) is combined with ETGBD, the orifice of the cystic duct in the common bile duct may be more easily detected in the cannulation procedure. The aim of this study was to evaluate the efficacy of ETGBD with IDUS compared with that of ETGBD alone.

Methods: A total of 100 consecutive patients with acute cholecystitis requiring ETGBD were retrospectively recruited. The first 50 consecutive patients were treated using ETGBD without IDUS, and the next 50 patients were treated using ETGBD with IDUS. Through propensity score matching analysis, we compared the clinical outcomes between the groups. The primary outcome was the technical success rate.

Results: The technical success rate of ETGBD with IDUS was significantly higher than that of ETGBD without IDUS (92.0\% vs. $76.0 \%, p=0.044)$. There was no significant difference in procedure length between the two groups ( $74.0 \mathrm{~min}$ vs. $66.7 \mathrm{~min}, p=0.310$ ). The complication rate of ETGBD with IDUS was significantly higher than that of ETGBD without IDUS (6.0\% vs. $0 \%$, $p<0.001)$; however, only one case showed an IDUS technique-related complication (pancreatitis).

Conclusions: The assistance of IDUS may be useful in ETGBD. Clin Endosc 2020;53:221-229

Key Words: Acute cholecystitis; Cystic duct; Endoscopic retrograde cholangiopancreatography; Endoscopic transpapillary gallbladder drainage; Intraductal ultrasonography

\section{INTRODUCTION}

Acute cholecystitis is one of the most common biliary diseases. Most patients with acute cholecystitis need urgent treatment and sometimes develop severe complications if appropriate drainage is not performed. Therefore, according to the Japanese guidelines, ${ }^{1}$ early gallbladder drainage is es-

Received: May 17, 2019 Revised: July 3, 2019

Accepted: July 24, 2019

Correspondence: Ryota Sagami

Department of Gastroenterology, New Tokyo Hospital, 1271 Wanagaya, Matsudo, Chiba 270-2232, Japan

Tel: +81-47-711-8700, Fax: +81-47-392-8718, E-mail: sagami1985@yahoo.co.jp ORCID: https://orcid.org/0000-0003-4961-3521

(c) This is an Open Access article distributed under the terms of the Creative Commons Attribution Non-Commercial License (http://creativecommons.org/ licenses/by-nc/3.0) which permits unrestricted non-commercial use, distribution, and reproduction in any medium, provided the original work is properly cited. sential in patients with severe (grade II or III) cholecystitis in the presence of multiple organ dysfunction and/or severe local inflammation. The gold standard therapeutic strategy is cholecystectomy; however, surgery may result in increased mortality, especially in elderly patients and/or in those with multiple severe comorbidities. ${ }^{2-7}$

The common drainage techniques include percutaneous transhepatic gallbladder drainage (PTGBD) and percutaneous transhepatic gallbladder aspiration (PTGBA), which are well known to be effective methods for primary drainage. - $^{8-12}$ However, these procedures cannot be adopted in patients with massive ascites, anatomically inaccessible gallbladder, ${ }^{13-15}$ risk of self-removal of the drainage tube, or bleeding tendency. ${ }^{16-18}$

Endoscopic transpapillary gallbladder drainage (ETGBD) is considered to be the second-line drainage procedure for acute cholecystitis. This method has also been reported to 
have a high success rate and a low complication rate, suggestive of a sufficient degree of efficacy in patients who are not candidates for PTGBD; ${ }^{9-23}$ however, some attention should be paid to acute pancreatitis as a representative complication. ${ }^{24}$ In addition, this method has a technical limitation and difficulty in that the cystic duct (CD) and the orifice of the $\mathrm{CD}$ cannot be enhanced during a cholangiography owing to tortuosity, calculus, or malignant obstruction of the $\mathrm{CD}^{25,26} \mathrm{In}$ a previous report, we proposed a new method of ETGBD combined with intraductal ultrasonography (IDUS). ${ }^{27}$ With the additional IDUS, the orifice of the $\mathrm{CD}$ in the common bile duct (CBD) can be easily detected and $\mathrm{CD}$ cannulation may also be easily performed. In the present study, we aimed to evaluate the efficacy and feasibility of ETGBD with IDUS compared with those of ETGBD alone, and to discuss whether this technique can be a standard method for gallbladder drainage in patients with acute cholecystitis.

\section{MATERIALS AND METHODS}

\section{Study design}

A total of 100 patients with acute cholecystitis requiring
ETGBD treatment from January 2015 to December 2017 were retrospectively enrolled in the present study. The first 50 consecutive patients out of the 100 patients were treated using ETGBD without IDUS and the next 50 consecutive patients were treated using ETGBD with IDUS. Acute cholecystitis was diagnosed according to the diagnostic criteria and classified into three grades ${ }^{1}$ according to severity and symptoms (Fig. 1).

Patients with grade III (severe) and grade II (moderate) acute cholecystitis required urgent drainage of the gallbladder. Grade I (mild) acute cholecystitis was defined as milder than grade III or II, and accordingly did not require drainage. However, we sometimes performed drainage in patients with grade I acute cholecystitis when their symptoms included intense abdominal pain and/or when they had a significantly severe inflammatory condition in which the C-reactive protein (CRP) level or white blood cell (WBC) count was 1.5 times higher on the second hospital day. ETGBD was first performed as an alternative to cholecystectomy or PTGBD if patients had massive ascites, anatomically inaccessible gallbladder, risk of self-removal of the drainage tube, and bleeding tendency, or if they had been administered oral antithrombotic agents.

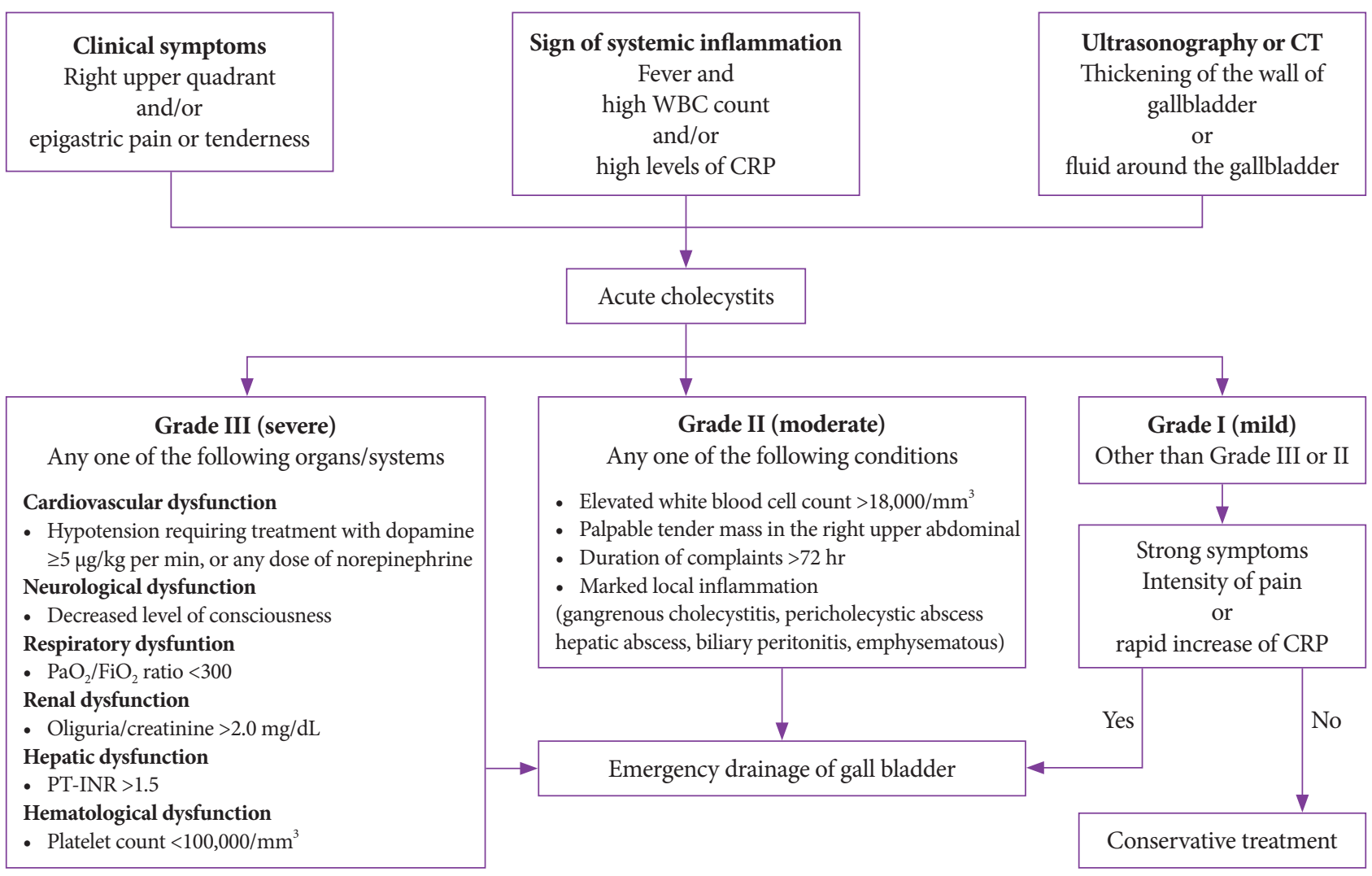

Fig. 1. Diagnostic procedure and classification criteria according to the severity grade of acute cholecystitis. Drainage is usually indicated for inoperable patients with grade III, grade II, or grade I (selected patients only) acute cholecystitis. CRP, C-reactive protein; CT, computed tomography; PT-INR, prothrombin time-international normalized ratio; WBC, white blood cell. 


\section{Propensity score matching}

We compared the usefulness of ETGBD with IDUS with that of ETGBD without IDUS in patients with acute cholecystitis by using propensity score matching to confirm the validity of this retrospective analysis. Propensity scores were calculated using logistic regression analysis. Age, sex, severity of acute cholecystitis, WBC count, CRP level, and presence of gallbladder stones were used as matching factors. After matching, univariate and multivariate logistic regression analyses were conducted. On the basis of the score obtained from each group, patients treated with ETGBD with or without IDUS were matched using calipers of width equal to 0.2 of the standard deviation of the logit of the propensity score.

\section{Techniques of ETGBD and ETGBD combined with IDUS}

ETGBD was performed using an endoscopic retrograde cholangiopancreatography catheter (MTW; ABIS, Tokyo, Japan) and a 0.025-inch guidewire (VisiGlide 2; Olympus Medical Systems, Tokyo, Japan). After CBD cannulation, endoscopic sphincterotomy or endoscopic papillary balloon dilation was performed. These treatments were considered in patients with a small ampulla of Vater in order to prevent postoperative cholangitis and/or pancreatitis. In the ETGBD without IDUS group, when the CD was imaged with contrast, attempts based on vague clues were made to insert the guide- wire into the gallbladder, and a 10-cm, 5- or 7-Fr pigtail stent was placed into the gallbladder. In the ETGBD with IDUS group, the orifice of the CD could be detected using IDUS with the portal vein as a landmark. First, the IDUS probe was inserted into the upper bile duct along a wire under X-ray guidance, and the portal vein was visualized on the IDUS image. While the IDUS probe was slowly pulled out from the upper bile duct to the papilla (Fig. 2A), the CD was detected around the $\mathrm{CBD}$ and the partition wall between the $\mathrm{CD}$ orifice and the CBD could be identified (Fig. 2B, C). Given that the IDUS image of the portal vein was adjusted to be at the 3 oclock position, the left side of the patient was revealed as the 3 oclock position and the dorsal side was the 12 oclock position (Fig. 2D). The position in which the partition wall became invisible on the IDUS image was the location of the CD branching point (orifice) from the CBD (Fig. 2E). The IDUS position was confirmed with radiography, and this could serve as a guide in performing CD cannulation (Fig. 2F). The procedure for inserting the drainage tube into the gallbladder was as mentioned above.

When ETGBD was a technical failure, PTGBD, PTGBA, endoscopic ultrasound-guided gallbladder drainage (EUSGBD), or elective cholecystectomy was performed.

\section{Outcomes and definitions}

First, we compared the technical success of gallbladder
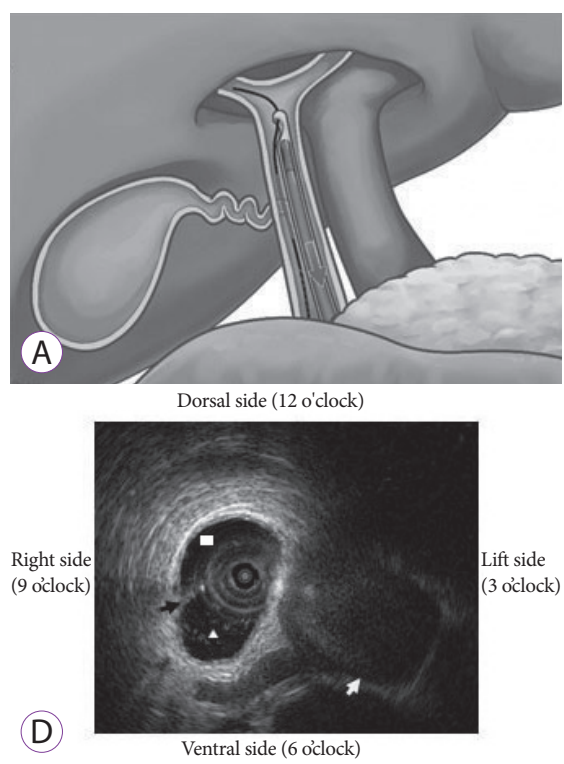
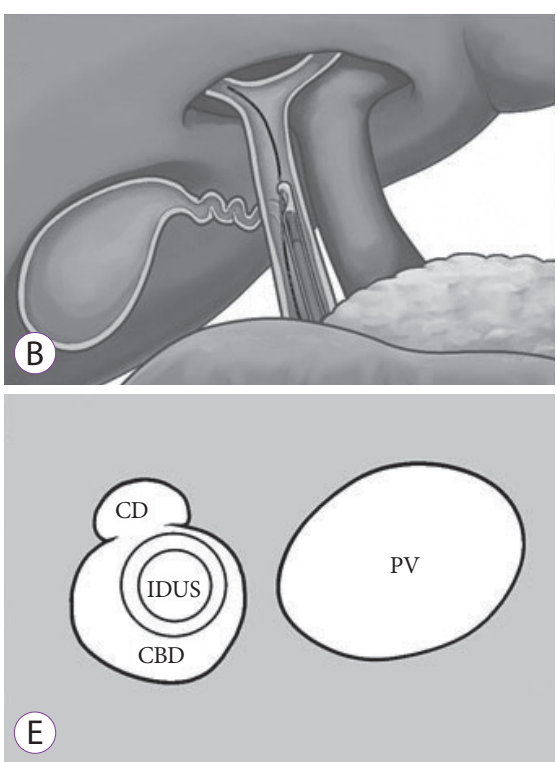
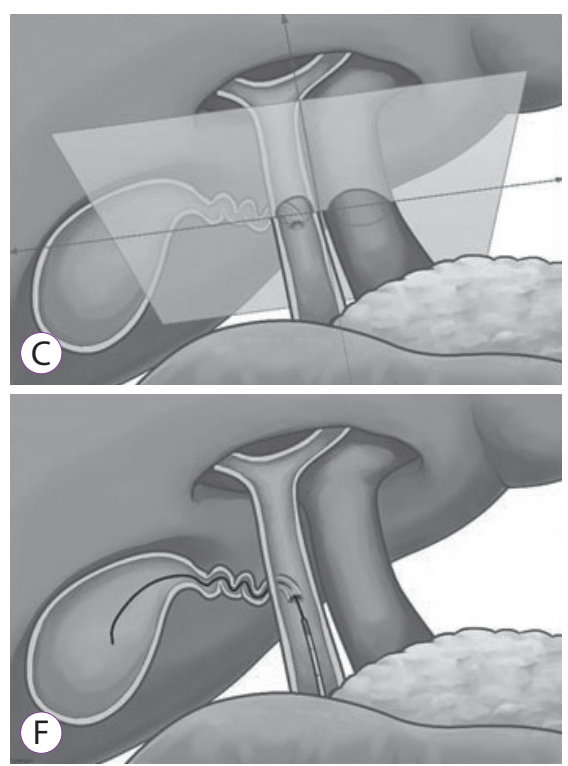

Fig. 2. Technique of endoscopic transpapillary gallbladder drainage with intraductal ultrasonography (IDUS). (A, B) The IDUS probe was slowly pulled out from the upper bile duct to the papilla. (C, D) The portal vein (PV) was adjusted to be at the 3 o'clock position. The left side of the patient was revealed as the 3 o'clock position and the dorsal side was the 12 o'clock position. The cystic duct (CD) was detected around the common bile duct (CBD). The orifice of the CD is shown by a white square. The lumen of the CBD is indicated by a white triangle. The partition wall between the orifice and the CBD is indicated by a black arrow, and the PV is shown by a white arrow. (E) The position in which the partition wall became invisible on the IDUS image was the location of the CD branching point (orifice) from the CBD. (F) $\mathrm{CD}$ cannulation was done with the guidewire. 
drainage, procedure length, and incidence of complications between ETGBD with IDUS and ETGBD without IDUS. Second, we analyzed the clinical efficacy, length of hospitalization, and recurrence rate in patients with acute cholecystitis treated with ETGBD as the first choice. The technical success rate was defined as the rate of successful placement of the catheter into the gallbladder. The procedure length was defined as the time from the first touch of the duodenal papilla to the withdrawal of the endoscope. Clinical efficacy was evaluated on the basis of the number of days requiring WBC normalization, 50\% reduction of the CRP level from its peak value, and the start of oral intake. Recurrence was judged to have occurred when acute cholecystitis recurred according to the above-described diagnostic criteria because of stent dysfunction. Early stent dysfunction was defined as stent dysfunction occurring within 14 days after the procedure, and late stent dysfunction was defined as that occurring after 15 days. The clinical success of ETGBD was defined as sufficient improvement of cholecystitis allowing the patient to leave the hospital without stent dysfunction or other complication.

Table 1. Univariate Analysis of Baseline Characteristics in Endoscopic Transpapillary Gallbladder Drainage without and with Intraductal Ultrasonography Groups

\begin{tabular}{|c|c|c|c|c|c|c|}
\hline & \multicolumn{3}{|c|}{ Before propensity score matching } & \multicolumn{3}{|c|}{ After propensity score matching } \\
\hline & $\begin{array}{l}\text { Without IDUS } \\
\text { group }(n=50)\end{array}$ & $\begin{array}{c}\text { With IDUS } \\
\text { group }(n=50)\end{array}$ & $p$-value & $\begin{array}{l}\text { Without IDUS } \\
\text { group }(n=42)\end{array}$ & $\begin{array}{c}\text { With IDUS } \\
\text { group }(n=50)\end{array}$ & $p$-value \\
\hline Mean age, yr (SD) & $72.7(12.3)$ & $73.5(11.0)$ & 0.739 & $74.4(11.3)$ & $73.5(11.0)$ & 0.697 \\
\hline Sex (Male) & $18(36 \%)$ & $18(36 \%)$ & 1.000 & $16(38 \%)$ & $18(36 \%)$ & 0.836 \\
\hline \multicolumn{7}{|l|}{ Severity grade of cholecystitis } \\
\hline Grade I (mild) & $21(42 \%)$ & $27(54 \%)$ & 0.375 & $21(50 \%)$ & $27(54 \%)$ & 0.472 \\
\hline Grade II (moderate) & $20(40 \%)$ & $18(36 \%)$ & & $14(33 \%)$ & $18(36 \%)$ & \\
\hline Grade III (severe) & $9(18 \%)$ & $5(10 \%)$ & & $7(17 \%)$ & $5(10 \%)$ & \\
\hline $\mathrm{WBC}$ mean $\pm \mathrm{SD}\left(\times 10^{3} / \mu \mathrm{L}\right)$ & $12.0 \pm 5.8$ & $11.9 \pm 6.6$ & 0.966 & $12.4 \pm 5.9$ & $11.9 \pm 6.6$ & 0.667 \\
\hline $\mathrm{CRP}$ mean $\pm \mathrm{SD}(\mathrm{mg} / \mathrm{dL})$ & $11.8 \pm 9.8$ & $11.5 \pm 11.0$ & 0.806 & $12.0 \pm 10.2$ & $11.5 \pm 11.0$ & 0.738 \\
\hline Antithrombotic agents & $25(50 \%)$ & $28(56 \%)$ & 0.548 & $23(55 \%)$ & $28(56 \%)$ & 0.905 \\
\hline \multicolumn{7}{|l|}{ Common bile duct stone } \\
\hline None & $25(50 \%)$ & $21(42 \%)$ & 0.839 & $21(50 \%)$ & $21(42 \%)$ & 0.847 \\
\hline Stone & $14(28 \%)$ & $18(36 \%)$ & & $10(24 \%)$ & $18(36 \%)$ & \\
\hline Sludge & $10(20 \%)$ & $10(20 \%)$ & & $10(24 \%)$ & $10(20 \%)$ & \\
\hline Cancer & $1(2 \%)$ & $1(2 \%)$ & & $1(2 \%)$ & $1(2 \%)$ & \\
\hline \multicolumn{7}{|l|}{ Gallbladder stone } \\
\hline None & $5(10 \%)$ & $5(10 \%)$ & 0.954 & $4(10 \%)$ & $5(10 \%)$ & 0.945 \\
\hline Stone & $38(76 \%)$ & $39(78 \%)$ & & $33(79 \%)$ & $39(78 \%)$ & \\
\hline Sludge & $5(10 \%)$ & $5(10 \%)$ & & $5(10 \%)$ & $5(10 \%)$ & \\
\hline Cancer & $2(4 \%)$ & $1(2 \%)$ & & $2(5 \%)$ & $1(2 \%)$ & \\
\hline
\end{tabular}

CRP, C-reactive protein; IDUS, intraductal ultrasonography; SD, standard deviation; WBC, white blood cell.

Table 2. Univariate Analysis of Procedure Related Factors in the Endoscopic Transpapillary Gallbladder Drainage without and with Intraductal Ultrasonography Groups (propensity score matching)

\begin{tabular}{lccc}
\hline & $\begin{array}{c}\text { Without IDUS group } \\
(\boldsymbol{n = 4 2 )}\end{array}$ & $\begin{array}{c}\text { With IDUS group } \\
(\boldsymbol{n}=\mathbf{5 0})\end{array}$ & $\boldsymbol{p}$-value \\
\hline Contrast of CD & $26(62 \%)$ & $27(54 \%)$ & 0.445 \\
Contrast of gallbladder & $14(33 \%)$ & $10(20 \%)$ & 0.150 \\
Technical success rate & $32(76 \%)$ & $46(92 \%)^{\mathrm{a})}$ & 0.044 \\
Procedure lengths (min) & $66.7(14-215)$ & $74(10-140)$ & 0.310 \\
Complication rate & $0(0 \%)$ & $3(6 \%) \mathrm{a})$ & $<0.001$ \\
\hline
\end{tabular}

CD, cystic duct; IDUS, intraductal ultrasonography. ${ }^{\text {a) }} p<0.05$. 
This retrospective study was approved by the Institutional Review Board of New Tokyo Hospital (approval no. 99), registered in the University Hospital Medical Network Clinical Trials Registry (UMIN 000035646), and conducted in accordance with the Declaration of Helsinki. Informed consent was obtained from each patient before enrollment.

\section{Statistical analysis}

Categorical variables were analyzed using Fisher's exact test or the $\chi^{2}$ test. Continuous variables were expressed as mean standard deviation and analyzed using Student's $t$-test or the Mann-Whitney $U$-test. Logistic regression analyses were performed using covariates (among those described above) that could potentially influence the success rate of ETGBD, and $p<0.05$ was considered statistically significant. All data analyses were conducted using SPSS version 24.0 (IBM Co., Armonk, NY, USA).

\section{RESULTS}

In the present study, we enrolled 100 patients with acute cholecystitis from January 2015 to December 2017. All of them were selected to undergo ETGBD because of use of antithrombotic therapy, dementia with a possible risk of self-removal of the tube, or a critical complication precluding surgery. Among these 100 patients, the first 50 consecutive patients were treated using ETGBD without IDUS and the next 50 consecutive patients were treated using ETGBD with IDUS. The clinical characteristics of the patients in both groups are

Table 3. Analysis of Patient Prognosis after Gallbladder Drainage in Endoscopic Transpapillary Gallbladder Drainage without and with Intraductal Ultrasonography Groups (propensity score matching)

\begin{tabular}{lccc}
\hline & $\begin{array}{c}\text { Without IDUS group } \\
(\boldsymbol{n}=\mathbf{4 2})\end{array}$ & $\begin{array}{c}\text { With IDUS group } \\
(\boldsymbol{n}=\mathbf{5 0})\end{array}$ & p-value \\
\hline Period until normalization of WBC (days) & $3.7(0-14)$ & $2.7(0-15)$ & 0.175 \\
Period until reduction by half from its peak of CRP (days) & $7.3(0-48)$ & $5.6(0-20)$ & 0.135 \\
Period until oral intake (days) & $5.4(2-23)$ & $4.7(2-17)$ & 0.336 \\
Length of hospitalization (days) & $10.2(3-48)$ & $8.3(3-23)$ & 0.074 \\
\hline
\end{tabular}

CRP, C-reactive protein; IDUS, intraductal ultrasonography; WBC, white blood cell.

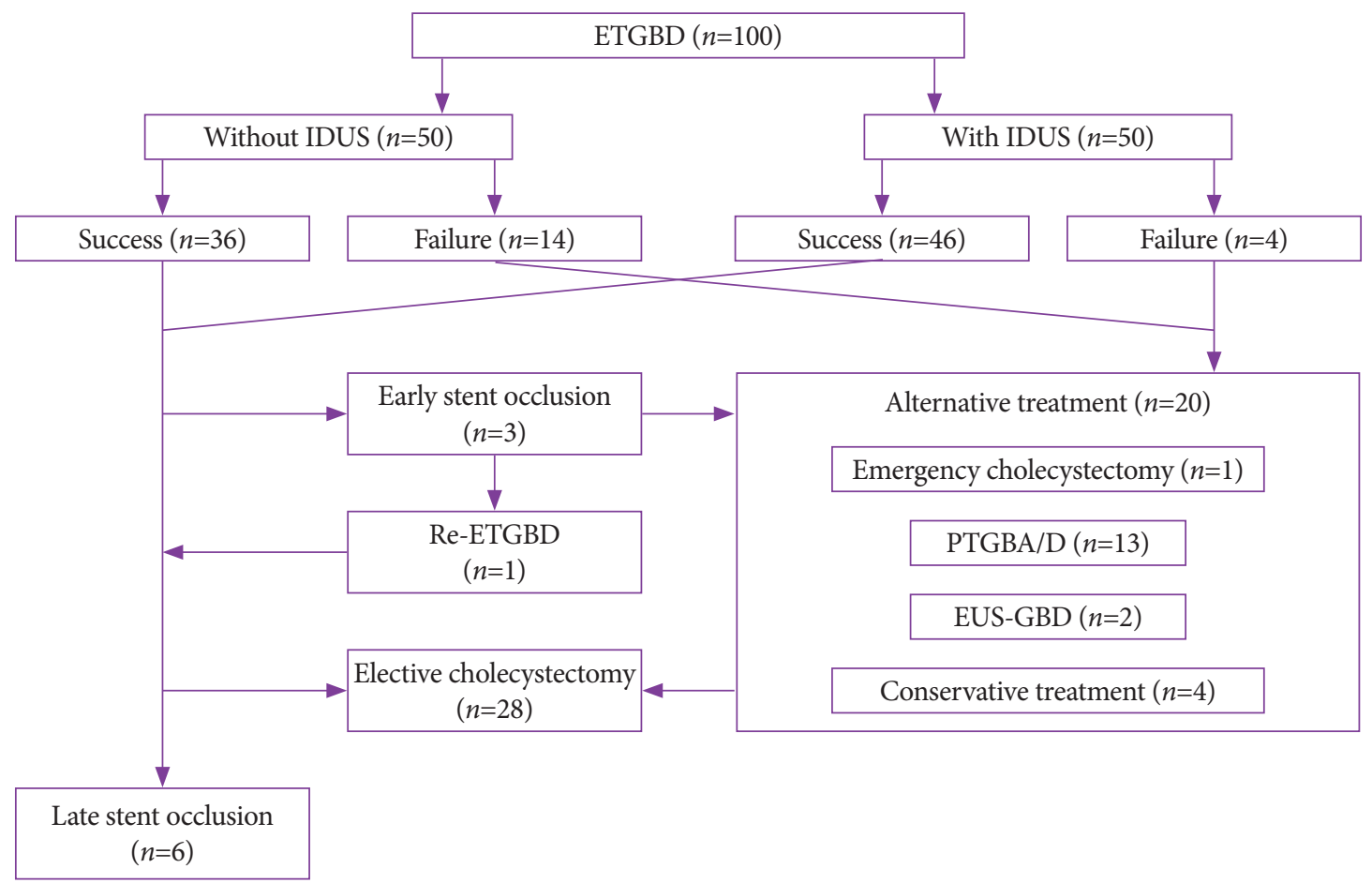

Fig. 3. Clinical courses of 100 patients with acute cholecystitis treated using endoscopic transpapillary gallbladder drainage (ETGBD). EUS-GBD, endoscopic ultrasound-guided gallbladder drainage; IDUS, intraductal ultrasonography; PTGBA/D, percutaneous transhepatic gallbladder aspiration/drainage. 
shown in Table 1. There were no significant differences in patient characteristics between the ETGBD with IDUS and ETGBD without IDUS groups. Matched pairs (42 patients from the ETGBD without IDUS group and 50 patients from the ETGBD with IDUS group) were analyzed after propensity score matching (Table 1). There were no significant differences in categorical data between the two groups.

A comparison of therapeutic outcomes between the ETGBD with IDUS and ETGBD without IDUS groups is shown in Table 2. The success rate of ETBGD with IDUS was significantly higher than that of ETBGD without IDUS (92\% [46/50] vs. $76 \%[32 / 42], p=0.044)$. There was no significant difference in the procedure length between the two groups (66.7 [14-215] min vs. 74 [10-140] min, $p=0.310$ ). The complication rate in the group with IDUS was significantly higher than that in the group without IDUS (6.0\% [3/50] vs. $0 \%$ [0/42], $p<0.001)$. These complications included mild pancreatitis, bleeding after endoscopic sphincterotomy, and small CD perforation caused by the guidewire. All patients with complications recovered with conservative treatment, and only one case of IDUS technique-related complication was observed. The clinical course after gallbladder drainage of patients in both groups is shown in Table 3. There was no significant difference in patient prognosis between the two groups.

The overall clinical results of ETGBD cases are shown in Fig. 3. The overall technical and clinical success rates of all ETGBD cases were 82\% (82/100) and 96\% (79/82), respectively. Early stent dysfunction was found in $3.7 \%$ of patients $(3 / 82 ; 1$ in the group with IDUS and 2 in the group without IDUS), and ETGBD was successfully re-tried in one patient. Patients who could not be successfully treated with ETGBD $(20.0 \%, 20 / 100)$ were alternatively treated using emergency cholecystectomy, PTGBA/PTGBD, EUS-GBD, or conservative treatment including the administration of antibacterial drugs while fasting. Elective cholecystectomy was performed in 28 patients on the next day after the removal of the ETGBD
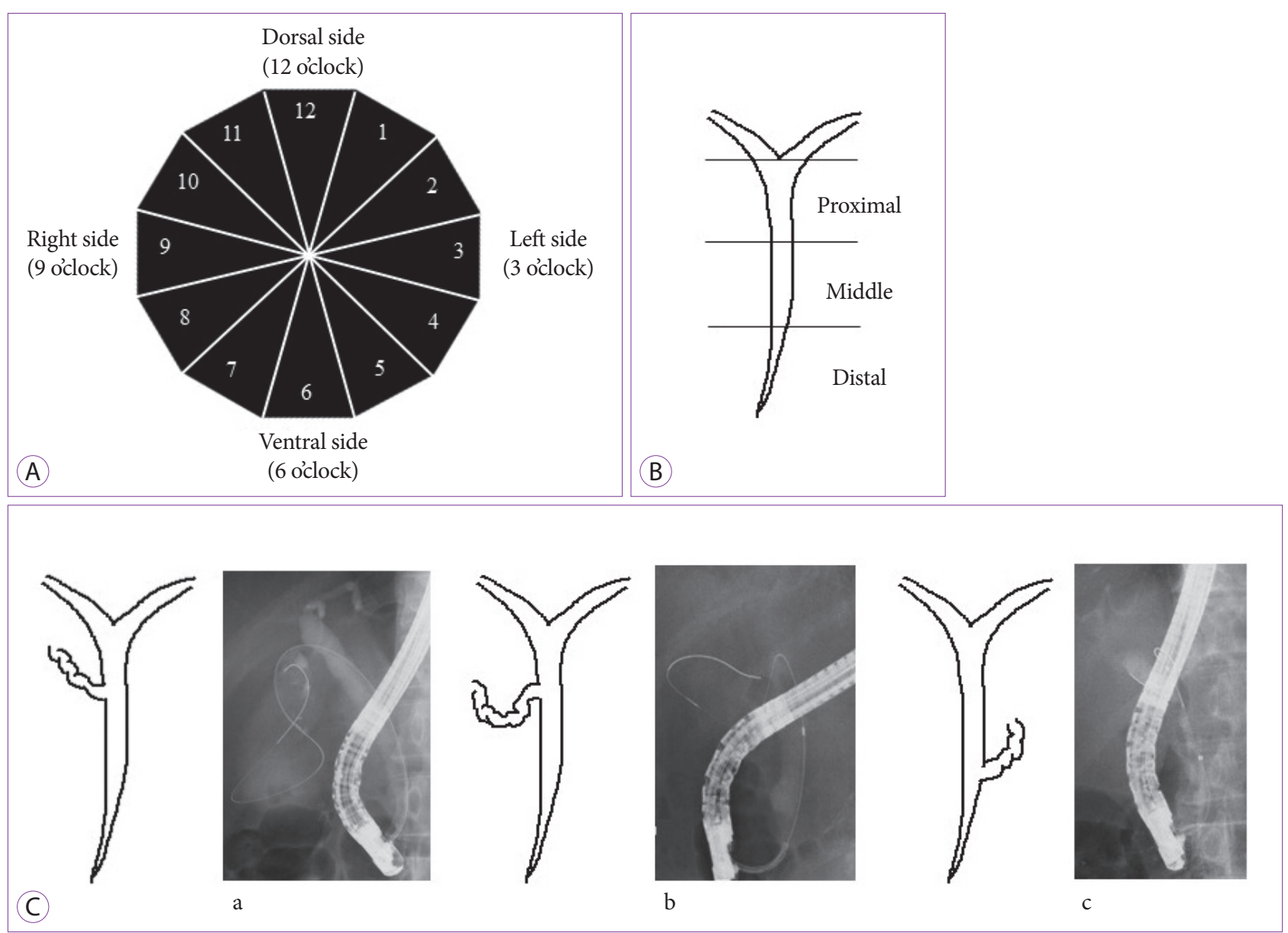

Fig. 4. Variations of the orifice of the cystic duct (CD) on intraductal ultrasonography (IDUS) examination. (A) The o'clock position of the direction of the CD orifice was determined using IDUS. The directions were described as in a clock face, with the common bile duct as the center, the dorsal side as the 12 o'clock direction, and the right side as the 9 o'clock direction. (B) The position of the bifurcation of the CD and extrahepatic bile duct was divided into the following 3 types: distal, middle, and proximal. (C) Three subgroups based on the angle of the orifice of the $\mathrm{CD}$ : a) right upper branch; b) right downward branch; and c) left upper branch. 
stent. Late stent dysfunction occurred in $7.3 \%$ of patients (6/82; 3 in the group with IDUS and 3 in the group without IDUS) after an average observation period of 176 (range, 55-337) days.

\section{DISCUSSION}

Several studies have reported that ETGBD has a technical success rate of $64 \%-100 \%$, a clinical success rate of $62 \%-100 \%$, and a complication rate of $0 \%-17.2 \%$, representing inter-institutional variances. ${ }^{19-23,28-31}$ ETGBD is considered to produce a better therapeutic effect than that of PTGBD especially in patients with massive ascites, anatomically inaccessible gallbladder, ${ }^{13-15}$ and a possible risk of self-removal of the tube. ${ }^{24}$ In addition, this method should be recommended to patients

Table 4. Relationship between Orifice of Cystic Duct and Success of Gallbladder Stent Placement

\begin{tabular}{|c|c|}
\hline & $\begin{array}{l}\text { Successful case of gallblad- } \\
\text { der stent placement, } n(\%)\end{array}$ \\
\hline \multicolumn{2}{|c|}{$\begin{array}{l}\text { Radial position of CD orifice } \\
\text { (o'clock direction) }\end{array}$} \\
\hline Dorsal side $(n=34)$ & $30(88.2)$ \\
\hline $11(n=20)$ & $18(90.0)$ \\
\hline $12(n=13)$ & $11(84.6)$ \\
\hline $1(n=1)$ & $1(100)$ \\
\hline Left side $(n=3)$ & $3(100)$ \\
\hline $2(n=2)$ & $2(100)$ \\
\hline $3(n=0)$ & $0(0)$ \\
\hline $4(n=1)$ & $1(100)$ \\
\hline Ventral side $(n=2)$ & $2(100)$ \\
\hline $5(n=0)$ & $0(0)$ \\
\hline $6(n=1)$ & $1(100)$ \\
\hline $7(n=1)$ & $1(100)$ \\
\hline Right side $(n=11)$ & $11(100)$ \\
\hline $8(n=1)$ & $1(100)$ \\
\hline $9(n=4)$ & $4(100)$ \\
\hline $10(n=6)$ & $6(100)$ \\
\hline \multicolumn{2}{|c|}{ Axial position of $\mathrm{CD}$ orifice } \\
\hline Proximal $(n=2)$ & $2(100)$ \\
\hline Middle $(n=43)$ & $39(90.7)$ \\
\hline Distal $(n=5)$ & $5(100)$ \\
\hline \multicolumn{2}{|l|}{ Direction of CD } \\
\hline RU branch $(n=43)$ & $40(93.0)$ \\
\hline RD branch $(n=2)$ & $2(100)$ \\
\hline LU branch $(n=5)$ & $4(80)$ \\
\hline
\end{tabular}

$\mathrm{CD}$, cystic duct; $\mathrm{LU}$, left upper; $\mathrm{RD}$, right downward; RU, right upper. with severe coagulopathy or those receiving antithrombotic therapy when PTGBD is contraindicated owing to a high risk of bleeding complications. ${ }^{16-18}$

ETGBD is technically challenging, especially the cannulation step and the passage of the guidewire through the $\mathrm{CD}$. More experience with wire manipulation possibly improves the technical success rate; ${ }^{29}$ however, the limitation of ETGBD is its technical difficulty that requires an expert endoscopist to negotiate the $\mathrm{CD}$, especially when the $\mathrm{CD}$ cannot be enhanced on a cholangiogram or when the guidewire cannot be inserted through the $\mathrm{CD}$ into the gallbladder because of tortuosity, steep angulation, calculus, or malignant obstruction. ${ }^{25,32}$ To aim for standardization of ETGBD, some special methods, combined with IDUS ${ }^{27}$ or cholangioscopy, ${ }^{33,34}$ have been previously proposed to make detecting the orifice of the $\mathrm{CD}$ in the $\mathrm{CBD}$ easier. In general, when the $\mathrm{CD}$ or gallbladder is not enhanced on a cholangiogram, $\mathrm{CD}$ cannulation becomes more difficult. ${ }^{25,32}$ However, our ETGBD combined with IDUS technique possibly improves the negotiation rate of the $\mathrm{CD}$ by considering the position of the $\mathrm{CD}$ branch level from the $\mathrm{CBD}$ even if the $\mathrm{CD}$ cannot be enhanced. Consequently, the technical success rate of ETGBD with IDUS was significantly higher than that of ETGBD without IDUS ( $92.0 \%$ vs. $76.0 \%$, $p=0.044$ ). This result suggests that the use of IDUS simplifies the negotiation of the $\mathrm{CD}$ orifice and does not require the assistance of duct contrast.

Although the procedure length of ETGBD with IDUS was expected to be longer than that of the conventional method, no statistically significant difference in procedure length was observed between the two groups. The complication rate of ETGBD with IDUS was significantly higher than that of the conventional method ( $6.0 \%$ vs. $0 \%, p<0.001)$. However, the complications were not serious and resolved quickly with conservative management. Only one case of pancreatitis was observed as an IDUS technique-related complication. In addition, the overall clinical success rate was high (96\%). Therefore, it seems that ETGBD with IDUS is a useful and highly effective procedure providing good therapeutic outcomes in patients with acute cholecystitis.

The present study has some limitations. First, the number of enrolled patients was relatively small, although it was not smaller than that of previous studies. Second, the results were possibly affected by the learning curve of the enrolled endoscopists. All ETGBD procedures were performed by two expert endoscopists with experience of $>50$ ETGBD cases. The procedure length was relatively long because not only the ETGBD procedure itself but also the treatment of the bile duct and pancreatic duct were included in the analysis in this study. The ETBGD procedures without and with IDUS were divided into the first 25 and last 25 cases, respectively, and the 
technical success rate and procedure length were evaluated in both groups. However, there were no statistically significant differences in these two parameters between the first and last 25 cases. Thus, both parameters were not considered to be affected by the learning curve of the endoscopists.

Third, this method requires some special endoscopic techniques owing to anatomical variations of the $\mathrm{CD}$ orifice. Such variations possibly make $\mathrm{CD}$ cannulation difficult. Therefore, to overcome this technical difficulty, we investigated the position of the $\mathrm{CD}$ orifice and the direction of the $\mathrm{CD}$ in this study. The oclock position of the direction of the $\mathrm{CD}$ orifice was determined in the IDUS image shown in Fig. 4A. The position of the bifurcation of the $\mathrm{CD}$ from the extrahepatic bile duct was divided into the following three types: distal, middle, and proximal (Fig. 4B). The CD orifice was also divided into three subgroups based on the location and angle of the orifice (Fig. 4C): right upper (RU) branch (Fig. 4C-a), right downward branch (Fig. 4C-b), and left upper (LU) branch (Fig. 4C-c) types. ${ }^{35-37}$

The most frequent branch types were dorsal $(11,12$, and 1 oclock positions; $68.0 \%, 34 / 50)$, middle $(86.0 \%, 43 / 50)$, and RU $(86.0 \%, 43 / 50)$. The success rates of gallbladder stent placement were $88 \%$ in the dorsal side, $90.7 \%$ in the middle, $93 \%$ in the RU branch, $80 \%$ in the $\mathrm{LU}$ branch, and $100 \%$ in the other side (Table 4). This information is considered to be helpful not only for facilitating the cannulation of the $\mathrm{CD}$ orifice but also for educating trainee endoscopists. Although this information might also aid $\mathrm{CD}$ cannulation, the number of analyzed cases may be too small to draw definite conclusions about the anatomical variations in the position of the $\mathrm{CD}$ orifice and the direction of the $\mathrm{CD}$. Further studies with larger numbers of patients are necessary.

In conclusion, ETGBD combined with IDUS is a feasible technique with a high success rate. IDUS may be a useful supportive method for ETGBD and its addition may aid in ETGBD standardization.

\section{Conflicts of Interest}

The authors have no financial conflicts of interest.

\section{Author Contributions}

Conceptualization: Ryota Sagami, Kenji Hayasaka

Data curation: RS, KH, Tetsuro Ujihara, Ryotaro Nakahara, Daisuke Murakami, Tomoyuki Iwaki, Satoshi Suehiro, Yasushi Katsuyama, Hideaki Harada, Yuji Amano

Formal analysis: RS, KH, TU, RN, DM, TI, SS, YK, HH, YA

Methodology: RS, KH

Project administration: YA

Supervision: YA

Writing-original draft: RS

Writing-review\&editing: YA

\section{REFERENCES}

1. Yokoe M, Hata J, Takada T, et al. Tokyo guidelines 2018: diagnostic criteria and severity grading of acute cholecystitis (with videos). J Hepatobiliary Pancreat Sci 2018;25:41-54.

2. Hannan EL, Imperato PJ, Nenner RP, Starr H. Laparoscopic and open cholecystectomy in New York state: mortality, complications, and choice of procedure. Surgery 1999;125:223-231.

3. Duncan CB, Riall TS. Evidence-based current surgical practice: calculous gallbladder disease. J Gastrointest Surg 2012;16:2011-2025.

4. Csikesz NG, Tseng JF, Shah SA. Trends in surgical management for acute cholecystitis. Surgery 2008;144:283-289.

5. Margiotta SJ Jr, Horwitz JR, Willis IH, Wallack MK. Cholecystectomy in the elderly. Am J Surg 1988;156:509-512.

6. Glenn F. Cholecystostomy in the high risk patient with biliary tract disease. Ann Surg 1977;185:185-191.

7. Roslyn JJ, Binns GS, Hughes EF, Saunders-Kirkwood K, Zinner MJ, Cates JA. Open cholecystectomy. A contemporary analysis of 42,474 patients. Ann Surg 1993;218:129-137.

8. Chopra S, Dodd GD 3rd, Mumbower AL, et al. Treatment of acute cholecystitis in non-critically ill patients at high surgical risk: comparison of clinical outcomes after gallbladder aspiration and after percutaneous cholecystostomy. AJR Am J Roentgenol 2001;176:1025-1031.

9. Ito K, Fujita N, Noda Y, et al. Percutaneous cholecystostomy versus gallbladder aspiration for acute cholecystitis: a prospective randomized controlled trial. AJR Am J Roentgenol 2004;183:193-196.

10. Sosna J, Copel L, Kane RA, Kruskal JB. Ultrasound-guided percutaneous cholecystostomy: update on technique and clinical applications. Surg Technol Int 2003;11:135-139.

11. Patel M, Miedema BW, James MA, Marshall JB. Percutaneous cholecystostomy is an effective treatment for high-risk patients with acute cholecystitis. Am Surg 2000;66:33-37.

12. Griniatsos J, Petrou A, Pappas P, et al. Percutaneous cholecystostomy without interval cholecystectomy as definitive treatment of acute cholecystitis in elderly and critically ill patients. South Med J 2008;101:586590.

13. Itoi T, Sofuni A, Itokawa F, et al. Endoscopic transpapillary gallbladder drainage in patients with acute cholecystitis in whom percutaneous transhepatic approach is contraindicated or anatomically impossible (with video). Gastrointest Endosc 2008;68:455-460.

14. Hasan MK, Itoi T, Varadarajulu S. Endoscopic management of acute cholecystitis. Gastrointest Endosc Clin N Am 2013;23:453-459.

15. Mori Y, Itoi T, Baron TH, et al. Tokyo guidelines 2018: management strategies for gallbladder drainage in patients with acute cholecystitis (with videos). J Hepatobiliary Pancreat Sci 2018;25:87-95.

16. Burke DR, Lewis CA, Cardella JF, et al. Quality improvement guidelines for percutaneous transhepatic cholangiography and biliary drainage. J Vasc Interv Radiol 2003;14(9 Pt 2):S243-S246.

17. Hatzidakis AA, Prassopoulos P, Petinarakis I, et al. Acute cholecystitis in high-risk patients: percutaneous cholecystostomy vs conservative treatment. Eur Radiol 2002;12:1778-1784.

18. Ha JP, Tsui KK, Tang CN, Siu WT, Fung KH, Li MK. Cholecystectomy or not after percutaneous cholecystostomy for acute calculous cholecystitis in high-risk patients. Hepatogastroenterology 2008;55:1497-1502.

19. Tamada K, Seki H, Sato K, et al. Efficacy of endoscopic retrograde cholecystoendoprosthesis (ERCCE) for cholecystitis. Endoscopy 1991;23:23.

20. Feretis C, Apostolidis N, Mallas E, Manouras A, Papadimitriou J. Endoscopic drainage of acute obstructive cholecystitis in patients with increased operative risk. Endoscopy 1993;25:392-395.

21. Kalloo AN, Thuluvath PJ, Pasricha PJ. Treatment of high-risk patients with symptomatic cholelithiasis by endoscopic gallbladder stenting. Gastrointest Endosc 1994;40:608-610.

22. Gaglio PJ, Buniak B, Leevy CB. Primary endoscopic retrograde chole- 
cystoendoprosthesis: a nonsurgical modality for symptomatic cholelithiasis in cirrhotic patients. Gastrointest Endosc 1996;44:339-342.

23. Itoi T, Coelho-Prabhu N, Baron TH. Endoscopic gallbladder drainage for management of acute cholecystitis. Gastrointest Endosc 2010;71:1038-1045.

24. Yu W, Li W, Wang Z, Ye X, Li N, Li J. Early percutaneous transhepatic gallbladder drainage compared with endoscopic retrograde cholangiopancreatography and papillotomy treatment for severe gallstone associated acute pancreatitis. Postgrad Med J 2007;83:187-191.

25. Widmer J, Alvarez P, Sharaiha RZ, et al. Endoscopic gallbladder drainage for acute cholecystitis. Clin Endosc 2015;48:411-420.

26. Súbtil JC, Betes M, Muñoz-Navas M. Gallbladder drainage guided by endoscopic ultrasound. World J Gastrointest Endosc 2010;2:203-209.

27. Hayasaka K, Harada H, Shimizu T, Suehiro S, Katsuyama Y. The effectiveness of intraductal ultrasonography for cystic duct cannulation. Gastrointest Endosc 2017;85:1307-1308.

28. Ogawa O, Yoshikumi H, Maruoka N, et al. Predicting the success of endoscopic transpapillary gallbladder drainage for patients with acute cholecystitis during pretreatment evaluation. Can J Gastroenterol 2008;22:681-685.

29. Kjaer DW, Kruse A, Funch-Jensen P. Endoscopic gallbladder drainage of patients with acute cholecystitis. Endoscopy 2007;39:304-308.

30. Pannala R, Petersen BT, Gostout CJ, Topazian MD, Levy MJ, Baron TH.
Endoscopic transpapillary gallbladder drainage: 10-year single center experience. Minerva Gastroenterol Dietol 2008;54:107-113.

31. Mutignani M, Iacopini F, Perri V, et al. Endoscopic gallbladder drainage for acute cholecystitis: technical and clinical results. Endoscopy 2009;41:539-546.

32. Khan MA, Atiq O, Kubiliun N, et al. Efficacy and safety of endoscopic gallbladder drainage in acute cholecystitis: is it better than percutaneous gallbladder drainage? Gastrointest Endosc 2017;85:76-87.e3.

33. McCarthy ST, Tujios S, Fontana RJ, et al. Endoscopic transpapillary gallbladder stent placement is safe and effective in high-risk patients without cirrhosis. Dig Dis Sci 2015;60:2516-2522.

34. Barkay O, Bucksot L, Sherman S. Endoscopic transpapillary gallbladder drainage with the SpyGlass cholangiopancreatoscopy system. Gastrointest Endosc 2009;70:1039-1040.

35. Shaw MJ, Dorsher PJ, Vennes JA. Cystic duct anatomy: an endoscopic perspective. Am J Gastroenterol 1993;88:2102-2106.

36. Berci G. Biliary ductal anatomy and anomalies. The role of intraoperative cholangiography during laparoscopic cholecystectomy. Surg Clin North Am 1992;72:1069-1075.

37. Itoi T, Sofuni A, Itokawa F, Kurihara T, Tsuchiya T, Niito T. Endoscopic transpapillary gallbladder drainage. Gastroenterological Endoscopy 2010;52:3337-3346. 